\title{
Use of Thermal Sensors for Fall Detection in a Simulated Toilet Environment
}

\author{
Satoshi Shirogane, Hitomi Takahashi, Kenji Murata, Satoshi Kido, Tomoya Miyasaka, \\ Tadafumi Saga, Shuhei Sakurai, Toyohiro Hamaguchi, Toshiaki Tanaka
}

\begin{abstract}
Falls are likely to occur in a toilet. However, people typically are alone when they use the toilet; thus, if a fall occurs, it may be difficult to ask for help. Therefore, we constructed a fall detection system using a thermal image sensor that cannot distinguish details, such as a face, and has high suitability for the toilet environment. In this study, we investigate the influence of the sensor position on the fall discrimination rate.

Sensors were installed at four locations and normal toilet movements and falling postures were recorded using eight healthy adults. Discrimination formulas were prepared using thermal images obtained for four subjects, and discrimination rates of falling postures were calculated for the remaining four subjects, which were then compared relative to sensor positions.

The highest discrimination rate $(95.7 \%)$ was obtained with the sensor installed in front of the toilet at the height of the body center of the subject sitting on the toilet. The lowest discrimination rate $(60.2 \%)$ was obtained with the sensor installed in front of the toilet and close to the ceiling with respect to the subject sitting on the toilet.

To detect falls using thermal imaging, sensor positions should be based on various factors, such as whether the entire body can be imaged relative to the falling posture without depending only on the height and direction or whether the difference between normal activity and falling posture is clear, including the environment of the toilet and the assumed fall situation.
\end{abstract}

Index Terms-Thermal sensors, fall detection, toilet, simulated environment.

\section{INTRODUCTION}

Elderly and handicapped people can fall performing normal activities [1], [2]. An elderly person who falls may require long-term care, and further repeated falling increases

This research was subsidized by the Saitama Prefecture Industry-University Collaborative R\&D Project Grant-in-Aid (Id. No. 169, representative: Satoshi Kido) in 2018.

Satoshi Shirogane, Department of Assistive Technology, Research Institute of National Rehabilitation Center for Persons with Disabilities, 4-1 Namiki, Tokorozawa, Saitama, Japan

Hitomi Takahashi, Department of Physical Therapy, Faculty of Health and Medical Welfare, Saitama Prefectural University, Saitama, Japan.

Kenji Murata, Department of Physical Therapy, Faculty of Health and Medical Welfare, Saitama Prefectural University, Saitama, Japan.

Satoshi Kido, Department of Physical Therapy, Faculty of Health and Medical Welfare, Saitama Prefectural University, Saitama, Japan.

Tomoya Miyasaka, Faculty of Physical Therapy, Faculty of Health Sciences, Hokkaido University of Science, Hokkaido, Japan.

Tadafumi Saga, Development Department, Kuki Factory, CHINO Corporation, Saitama, Japan.

Shuhei Sakurai, Aoki Central Clinic, Saitama, Japan.

Toyohiro Hamaguchi, Department of Occupational Therapy, Faculty of Health and Medical Welfare, Saitama Prefectural University, Saitama, Japan.

Toshiaki Tanaka, Institute of Gerontology, The University of Tokyo, Tokyo, Japan, Department of Physical Therapy, Faculty of Health Sciences, Hokkaido University of Science, Sapporo, Hokkaido, Japan. the risk of death [3]. It has been reported that falls occur frequently in bathrooms and toilets. For example, Stevens et al. [4] analyzed the data of a representative emergency department in the United States and found that, for people older than 65 years, injuries occurring in bathrooms accounted for $2.5 \%$ of all unintended injuries, and approximately $80 \%$ of the people said that the injuries were the result of a fall. Another study of theirs [5] investigated 328 people 65 or older of local residence and found that, particularly for those who experienced injuries, $17.3 \%$ of falls occurred in the bathroom. Hill et al. [6] studied 138 people of advanced age who experienced a fall within six months after leaving the hospital and reported that the fall rate in the bathroom was $14.5 \%$, second to the bedroom. Bleijlevens et al. [7] studied 333 elderly people who were treated in an emergency room and reported that approximately $10 \%$ of the fall-related fractures occurred in the toilet. Various countermeasures have been proposed to mitigate the serious problem of falling in the bathroom; however, to date, the incidence rate has not been sufficiently reduced [8].

Typically, people prefer to relieve themselves privately. Thus, it may be difficult to ask for help in the case of a fall [9]. To ensure the safety of elderly people and persons with disabilities, it is particularly important to detect the fall quickly and to inform their caregivers.

Sensing technology can be applied to detect falls, and various systems have been developed [10], [11]. Typically, toilets are installed in a small room and the position of a person using the toilet is also limited. Thus, a video camera could be used to detect falls. However, as a person's private parts are exposed when using the toilet, people typically expect privacy and are averse to being monitored. Thus, handling recorded information requires particular consideration [12]. Fall sensing methods that employ video cameras do not consider the importance of privacy, and it becomes difficult to obtain permission to use such systems. Additionally, subjects will find such systems even more unacceptable because the captured video is stored for a certain period of time.

A thermal image sensor can detect the surface temperature of a body in a non-contact manner and can record it in the form of two-dimensional temperature data. Additionally, with thermal image sensing, it is possible to continuously record a person's movements, e.g., sitting, standing, and walking [13]. Furthermore, temperature data does not reveal identifying characteristics such as facial features; thus, privacy can be maintained without requiring special image processing. Previously, our research group analyzed how 10 
healthy adults moved in a bathroom when using the toilet and obtained a fall discrimination rate of $97.8 \%$ [14]. Thereafter, further improvements were made to achieve a fall discrimination rate of $99.5 \%$ [15]. However, for use in actual toilets, the discrimination rate is very likely to be affected by the position of the sensor. Therefore, we further investigated the difference in discrimination rate relative to the position of the sensor.

In this study, our objective was to obtain reference information regarding the selection of appropriate sensor positions to evaluate a fall detection system equipped with thermal image sensors in an actual environment. Note that we focused on the influence of the height and direction (front or side) of the sensors. Eight healthy volunteers participated in this study, and only four sensor locations were investigated; therefore the results obtained cannot be generalized for the elderly and disabled with respect to all possible sensor positions. However, the results can be used to select candidate installation positions for on-site trials and are expected to be useful for future related research.

\section{METHOD}

Initially, we simulated a subject's movement and falling motion relative to use of the toilet in a bathroom with installed sensors and recorded the subject's state. Next, using the obtained data, we performed a two-stage analysis. The first stage involved establishing a fall detection discrimination formula. Following the process established in our previous study [15], a discriminant was created using linear discriminant analysis with the data from half the subjects [15]. Next, using the obtained formula, the discrimination rate was obtained using the remaining half of the subject data. Based on this discrimination rate, we analyzed the impact of the position of the thermal image sensors. The thermal image sensor, the experimental environment, the subjects, and the analytical method are described below.

\section{A. Thermal image sensor and measurement environment}

Herein, we used TP-H0260AN (Chino Co., Tokyo, Japan) [16] thermal image sensors (Fig. 1, Temperature measurement range: $-20^{\circ} \mathrm{C}-300^{\circ} \mathrm{C}$, resolution: $0.5^{\circ} \mathrm{C}$, accuracy: $\pm 2 \%$ of the data, view angle: $60^{\circ}$, frame time: $6 \mathrm{~Hz}$, data points: $2256(47 \times 48))$. As long as the sensor is installed in a toilet of size that allows the use of a wheelchair, this device can identify a subject's posture because it senses the entire body. Moreover, TP-H0260AN sensor are relatively inexpensive (i.e., approximately $\$ 2,000$ per unit) than conventional products.

The experimental environment in the toilet room is outlined in Fig. 2. The sensors were installed in four locations, i.e., to the front and right side walls of the toilet at a height of $800 \mathrm{~mm}$ and $2280 \mathrm{~mm}$ from the floor, respectively (Fig. 2a and 2b). Additionally, to simulate the position of a falling posture, five marks were attached to the floor surface at arbitrary positions away from the toilet seat (Fig. 2c, marked with stars). Three of the marks were approximately $40 \mathrm{~cm}$ in three different directions away from the toilet seat starting from the vertex of the arc on the near side of the toilet seat. One mark was $40 \mathrm{~cm}$ from the center of the washbowl on the wall on the left side of the toilet seat, and the other mark was $40 \mathrm{~cm}$ from the center of the entrance on the wall in front of the toilet seat.
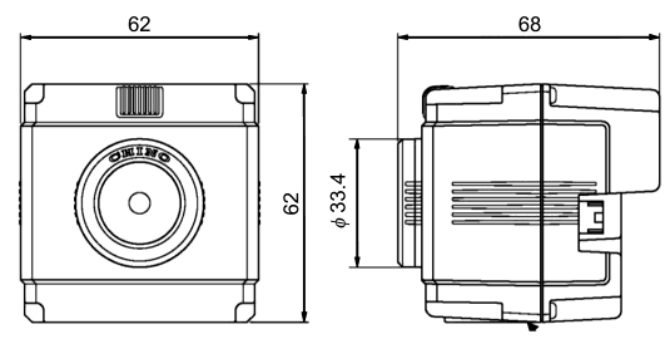

Fig. 1 Thermal image sensor unit TP-H0260AN

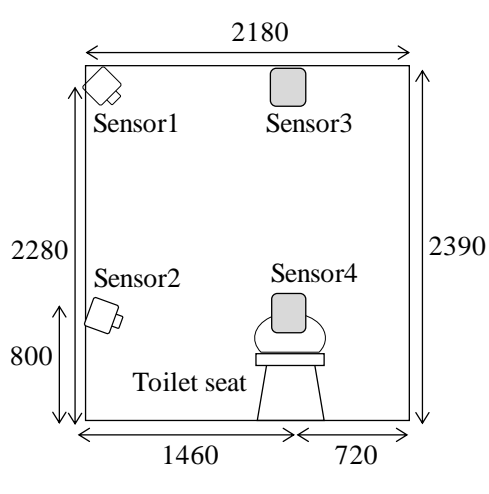

a.

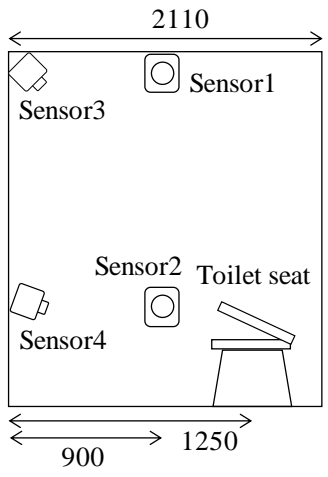

b.

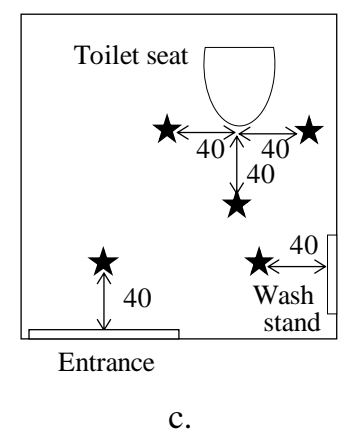

Fig. 2 Experimental environment

From left to right, a: front view, b: lateral view (from right), c: top view. The unit of the number is $\mathrm{mm}$. The star in the top view shows the simulated falling position.

\section{B. Collection of thermal image data}

The subjects were eight healthy adults (two men, six women, average age 21.5 years, average height $1.63 \mathrm{~m}$, average weight $56.1 \mathrm{~kg}$ ). A sufficient explanation of the research was orally provided and in writing in advance, and consent was obtained. Note that this research plan was approved by the Saitama Prefectural University Ethics Review Committee (approval number 29032).

In the toilet (Fig. 2) with the installed thermal image sensors, subjects imitated normal movements in the bathroom (Table 1) three times, and assumed 14 different falling 
patterns according to instructions provided by the examiner. The falling posture was assumed at the five positions marked on the floor. Here, the dorsal position, where the head was tilted to $0^{\circ}, 45^{\circ}$ and $90^{\circ}$ with respect to the toilet seat was captured. Note that the mark $40 \mathrm{~cm}$ to the left side of the toilet seat was excluded because, at that mark, it was impossible to assume a position where the trunk was tilted $90^{\circ}$ with respect to the toilet seat. These motions and attitudes were recorded using a thermal image sensor (sampling rate $1 \mathrm{~Hz}$ ).

Table 1 Simulated normal toilet operation

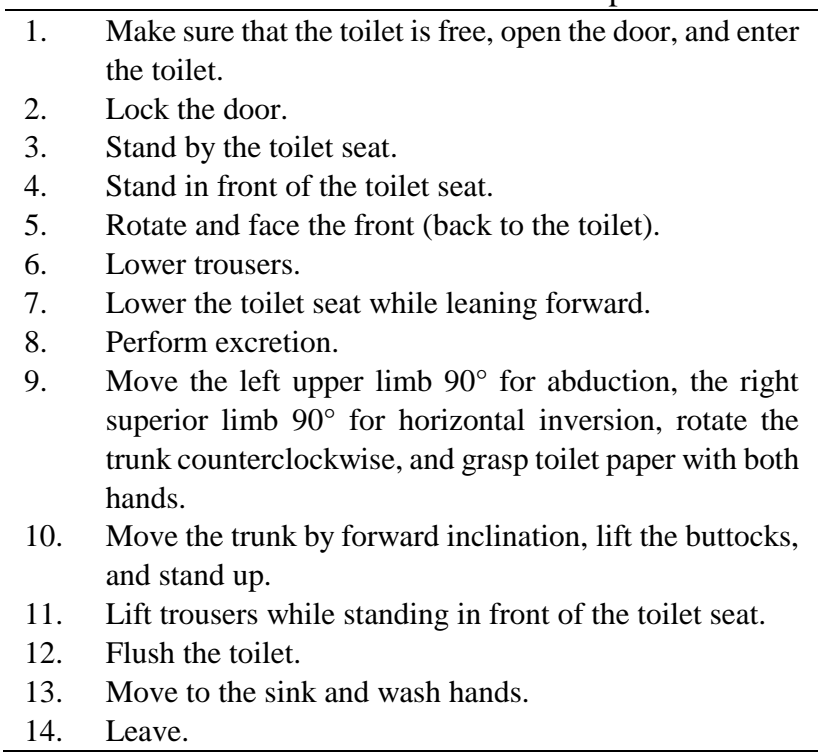

\section{Analytical method}

The obtained thermal image data were divided into normal operation data and fall data, and a discriminant formula was created using the data from four subjects. Then, 2256 data points were averaged and reduced to 552 points ( 23 points in length $\times 24$ points in width), and the discriminant formula (Fig. 3) was obtained by applying the discriminant analysis software used by Miyasaka et al. [15]. In the formula, $x n$ is an averaged temperature value, an is temperature coefficient, and $\mathrm{C}$ is the intercept. Using this formula, the discrimination rate and the error discrimination rate at each sensor position were calculated using the data from the remaining four subjects.

$$
\begin{gathered}
Z=a_{0} X_{0}+\underset{1}{a_{1} X_{1}}+\ldots+a_{550} X_{550}+a_{551} X_{551}+C \\
\text { to determine } A \text { (normal) when } Z<0
\end{gathered}
$$

Fig. 3 Discriminant formula to determine A (normal) when $\mathrm{Z}<0$

\section{RESULTS}

\section{A. Conformity of the discriminant formula created from data for four cases}

The discrimination rate for each sensor position is shown in Table 2. In the table, the upper row is the result based on the discriminant formula created using data for four cases. The "fall data discrimination rate" at each sensor position showed high values exceeding 98\% each. Similarly, for "normal data discrimination rate," i.e., normal toilet operation, values exceeding $98 \%$ were realized. The "lost info rate," which is the residual part of the probability, where discrimination failed despite being a falling state and the "false alarm rate," which is erroneously determined to be falling even though it is a normal operation were less than $2 \%$ at all sensor positions.

Table 2 Results of thermal image data analysis (discrimination rate)

\begin{tabular}{|c|c|c|c|}
\hline Dataset & Sensor & $\begin{array}{l}\text { Fall data } \\
\text { discrimination } \\
\text { rate } \\
\quad(\text { lost info } \\
\text { rate })[\%]\end{array}$ & $\begin{array}{l}\text { Normal data } \\
\text { discrimination } \\
\text { rate } \\
\quad \text { (false } \\
\text { alarm rate) } \\
{[\%]}\end{array}$ \\
\hline \multirow{4}{*}{$\begin{array}{l}\text { Data for } \\
\text { discriminant } \\
\text { formula } \\
\text { creation } \\
\text { (4 cases, } \\
8891 \text { data)* }\end{array}$} & $\begin{array}{l}\text { Sensor 1 } \\
\text { (lateral/upper) }\end{array}$ & 98.1 (1.9) & $99.4(0.6)$ \\
\hline & $\begin{array}{l}\text { Sensor } 2 \\
\text { (lateral/lower) }\end{array}$ & $100.0(0.0)$ & $100.0(0.0)$ \\
\hline & $\begin{array}{l}\text { Sensor } 3 \\
\text { (frontal/upper) }\end{array}$ & $99.8(0.2)$ & $98.6(1.4)$ \\
\hline & $\begin{array}{l}\text { Sensor } 4 \\
\text { (frontal/lower) }\end{array}$ & $99.1(0.9)$ & $99.9(0.1)$ \\
\hline \multirow{4}{*}{$\begin{array}{l}\text { Verification } \\
\text { data } \\
(4 \text { cases, } \\
12004 \\
\text { data)* }\end{array}$} & $\begin{array}{l}\text { Sensor 1 } \\
\text { (lateral/upper) }\end{array}$ & $95.2(4.8)$ & $93.2(6.8)$ \\
\hline & $\begin{array}{l}\text { Sensor } 2 \\
\text { (lateral/lower) }\end{array}$ & $60.2(39.8)$ & $99.1(0.9)$ \\
\hline & $\begin{array}{l}\text { Sensor } 3 \\
\text { (frontal/upper) }\end{array}$ & $85.7(14.3)$ & $95.1(4.9)$ \\
\hline & $\begin{array}{l}\text { Sensor } 4 \\
\text { (frontal/lower) }\end{array}$ & $95.7(4.3)$ & $98.8(1.2)$ \\
\hline
\end{tabular}

* Since the data recording time for each operation differs, the number of discrimination formula creation data and the number of verification data also differ. The lost info is the rate at which it is determined that if a case that should be judged as a fall is considered normal, and the false alarm rate is the rate at which normal movement is considered a fall.

\section{B. Conformity of discriminant formula generation to the data of the remaining four cases}

The results from analyzing the data of the remaining four cases using this discriminant formula are shown in the lower part of Table 2. For sensors 1 and 4, the fall rate was greater than 95\%; however, for sensors 3 and 2, it decreased significantly, i.e., $85.7 \%$ and $60.2 \%$, respectively. This decrease was particularly noticeable in sensor 2 . Regarding the judgment of normal operation, a discrimination rate exceeding $95 \%$ was demonstrated at all sensor positions.

\section{Typical thermal image obtained from installed sensors}

Fig. 4 displays an image of a typical normal operation and falling posture recorded by sensor 2 and a normal standing operation example recorded by sensors 1 and 3. Fig. 4(a) shows an image of a subject seated on a toilet seat captured from the side. Fig. 4(b) shows a subject standing upright in front of the toilet seat. Fig. 4(c) and 4(d) show subjects simulating a dorsal position on the floor after having fallen over or fallen on the floor. Fig. 4(e) and 4(f) show the 
standing posture of the subject during the toilet movement captured from different sensor positions.
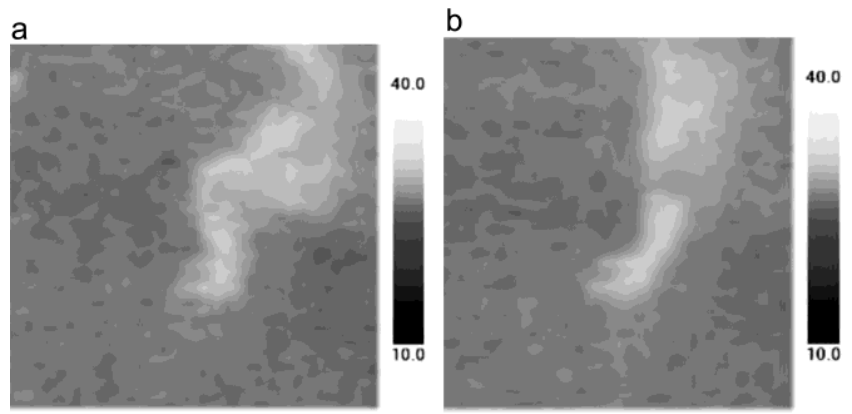

C

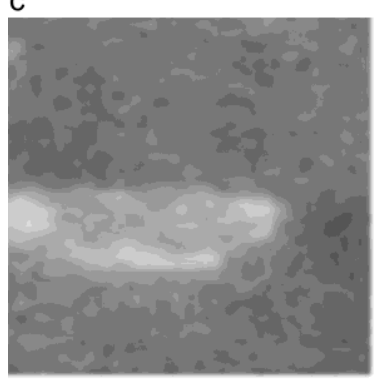

d
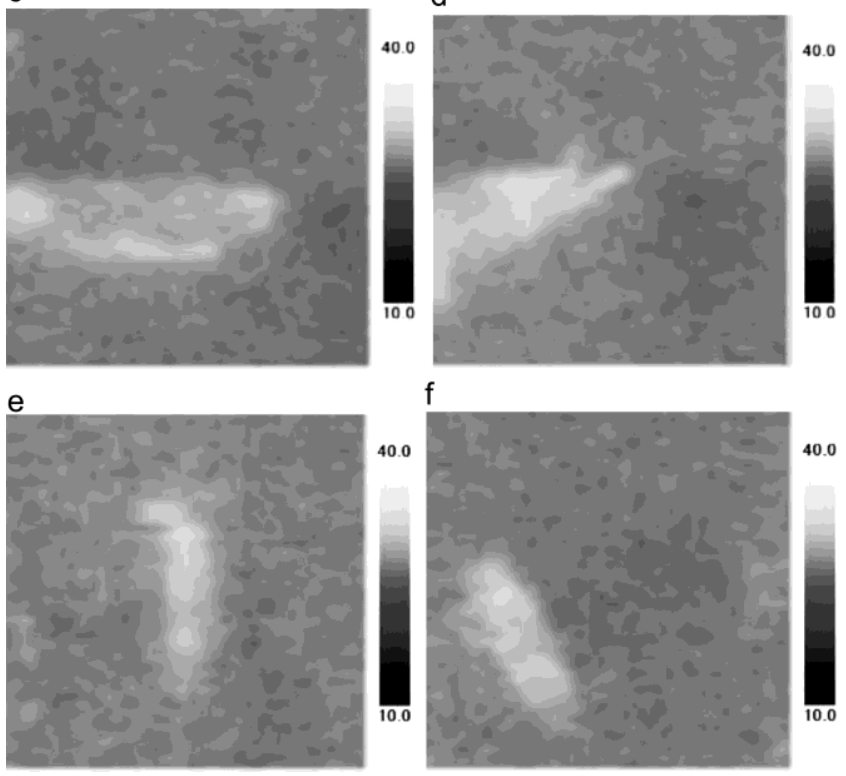

Fig. 4 Typical examples of recorded thermal image (a), (b) Example of normal toilet operation captured by sensor 2. (c), (d) Example of falling attitude captured by sensor 2. (e) Comparison of toilet actions captured by sensor 1 (e) and sensor 3 (f).

\section{DISCUSSION}

\section{A. Validity of discriminant formula}

Regarding the data used to create the discriminant formula, a high discrimination rate of $98 \%$ or greater was obtained for all sensors. The obtained results were comparable to those of previous studies by Kido et al. [14] and Miyasaka et al. [15]. Therefore, herein, we consider that the creation of the discriminant formula was properly implemented.

\section{B. Influence of thermal image sensor position on discrimination rate}

From the discrimination results of the remaining four cases obtained using the created discrimination formula, herein, we examine the impact of thermal image sensor position on discrimination rate. In the detection of the fall movement, the fall movement discrimination rate at sensors 1 and 4 was high and low at sensors 2 and 3. Sensor 2 was installed at a low position; thus, the head of the subject could not be captured by the sensor while the subject was standing (Fig. 4(b)). Additionally, there were cases (Fig. 4(c)) where the subject's lower limb was hidden behind the toilet seat when assuming a fallen posture at the back of the toilet seat. Furthermore, there were cases where the subject's entire body was not captured by the sensor because the distance between the sensor and subject was short (Fig. 4(d)). Similarly, sensor 4 (low position) did not detect the subject's head even though it was not hidden behind the toilet seat. Sensors 1 and 3 were installed at a high position; thus, it was possible to capture the subject's entire body. However, with these two sensors, the so-called false alarm rate that discriminates the falling posture as normal was as high as approximately 5-7\%. If the position of the sensor is high, it may be difficult to detect the difference between the dorsal position (a falling posture) and the upright position in normal operation. Since the thermal image sensor records an image in only two dimensions, accurately determine a three-dimensional posture using only these data is difficult.

\section{Ideal sensor positions}

The obtained results indicate that the sensor installation position for detecting a fall using a thermal image sensor was optimal when the sensor was facing the subject sitting on the toilet seat from the front at the same height as the center of the subject's body. The next favorable installation position was near the ceiling close to the side of the subject sitting on the toilet seat. Contrastingly, front installation close to the ceiling relative to the subject sitting on the toilet seat did not yield satisfactory results. These results demonstrate the effectiveness of the four selected places, they do not depend on only height and direction; thus, it is necessary to consider each environment and each fall situation, such as whether the whole body can be captured against the fall posture or whether the difference between normal actions and the fall posture is clear. Furthermore, according to the image confirmed, it is understood that the condition to be particularly considered to check sensor installation position is that if the position enables the capture of the whole body of the target. Structural constraints are expected in real-world applications; thus, the installation position must be considered according to the given environment. However, we expect that the present results can likely serve as valuable reference.

\section{Limitations}

The goal of this research was to identify sensor installation positions suitable for fall detection using thermal image sensors; however, the sensor positions were limited to four arbitrarily selected locations and the number of targets was limited to eight healthy adults. Thus, further verification is required to obtain more general results. Furthermore, this study targeted only healthy subjects and the assumed motions and postures were simulated according to examiner instruction; thus, there was no way to consider other situations. Moreover, we could not consider the characteristics of disabled people with high fall risks, such as hemiplegic persons, stroke patients, or wheelchair users. Particularly, it is expected that a wheelchair may block the sensor. Here, a situation may occur where part of the subject's body cannot be captured by the sensor. Thus, additional confirmation is necessary. 


\section{CONCLUSIONS}

In this study, we investigate the influence of sensor position on the fall discrimination rate. To this end, we have conducted experiments that involved eight healthy participants wherein sensors were installed at four positions, discrimination formulas were derived, and discrimination rates were calculated and compared relative to sensor positions.

We found that the discrimination rate may not depend on only the height and direction of the sensor. In other words, we found that the sensor position should consider the indoor environment of the toilet and the assumed falling circumstances. Additionally, other factors such as, whether the subject's entire body can be imaged in the falling posture and whether the difference between normal activity and the fallen posture can be clearly determined. In future, we intend to investigate sensor positions in hospital toilets and health care facilities for people of advanced age.

\section{ACKNOWLEDGMENT}

The authors thank the subjects for their participation.

\section{REFERENCES}

[1] Krauss, M.J., Nguyen, S.L., Dunagan, W.C., Birge, S., Costantinou, E., Johnson, S., et al., "Circumstances of patient falls and injuries in 9 hospitals in a Midwestern healthcare system," Infection Control \& Hospital Epidemiology, vol.28(5), 2007, pp.544-550.

[2] Suzuki, T., Sonoda, S., Saitoh, E., Murata, M., Shimizu, Y., \& Misawa, $\mathrm{K}$, "Incidence and consequence of falls among stroke rehabilitation inpatients during the recovery phase in relation to ADL," The Japanese Journal of Rehabilitation Medicine, vol.43, 2006, pp.180-185.

[3] Donald I.P., \& Bulpitt C. J., "The prognosis of falls in elderly people living at home," Age Ageing, vol.28(2), 1999, pp.121-125.

[4] Stevens J.A., Haas E.N, Haileyesus T., "Nonfatal bathroom injuries among persons aged $\geq 15$ years--United States, 2008," Journal of Safety Research, vol.42(4), 2011, pp.311-315.

[5] Stevens J.A, Mahoney J.E., \& Ehrenreich H., "Circumstances and outcomes of falls among high risk community-dwelling older adults," Injury epidemiology, vol.20, 2014, pp.1-5.

[6] Hill A.M., Hoffmann T, \& Haines T.P., "Circumstances of falls and falls-related injuries in a cohort of older patients following hospital discharge," Clinical Interventions in Aging, vol.8, 2013, pp.765-774.

[7] Bleijlevens M.H., Diederiks J.P., Hendriks M.R., van Haastregt J.C., Crebolder H.F., \& van Eijk J.T., "Relationship between location and activity in injurious falls: an exploratory study," BMC Geriatrics, vol.10(1), 2010, article no.40.

[8] Buzink S.N., Molenbroek J.F.M., Haagsman E.M., \& Bruin de R. "Falls in the toilet environment: a study on influential factors," Gerontechnology, vol.4(1), 2005, pp.15-26.

[9] Kawano Y., "A case study of falling situations and individual factors for falls among nursing home residents with short term prospective design," Journal of Japanese Society for Dementia Care, vol.6, 2007, pp.59-68. [Japanese]

[10] Mubashir M., Shao L., Seed L., "A survey on fall detection: Principles and approaches," Neurocomputing, vol.100, 2013, pp.144-152.

[11] Xu T., Zhou Y., \& Zhu J., "New advances and challenges of fall detection systems: a survey," Applied Sciences, vol.8(3), 2018, article no.418.

[12] Demiris G., Oliver D.P., Giger J., Skubic M., \& Rantz M., “Older adults' privacy considerations for vision based recognition methods of eldercare applications," Technology and Health Care, vol.17(1), 2009, pp.41-48.

[13] Iwasawa, S., Ebihara, K., Ohya, J., \& Morishima, S., "Real-time estimation of human body posture from monocular thermal images," Proceedings of IEEE Computer Society Conference on Computer Vision and Pattern Recognition, 1997, pp.15-20.

[14] Kido S., Miyasaka T., Tanaka T., Shimizu T., \& Saga T., "Fall detection in toilet rooms using thermal imaging sensors," Proceedings of 2009 IEEE/SICE International Symposium on System Integration (SII2009), 2009, pp.83-88

[15] Miyasaka T., Kido S., Saga T., \& Tanaka T., "Toilet activity fall detection employing discriminant analysis with two-dimensional temperature data as variables," GCBME2014/APCMBE2014, 2014 Paper ID: C3-0064-1.

[16] Shimizu T., Saga T., "The thermal imaging sensor with element of about 2000 pixels and applications," Proceedings of Technical Meeting on Sensors and Micromachines, PHS-09-25, 2009, pp.1-5.

Satoshi Shirrogane is a Chief of Clinical Evaluation Section, Department of Assistive Technology, Research Institute of National Rehabilitation Center for Persons with Disabilities in Japan. He is a physical therapist and has a Ph.D. (Physical Therapy), and a B.S. (Mechanical Engineering). He is interested in the clinical evaluation of assistive devices, working on the methodology and practice of various device evaluations.

Assictant Profesor, Kenji Murata,Ph.D.(1986). Department of Physical Therapy, Faculty of Health and Medical Welfare, Saitama Prefectural University. He obtained his doctorate in the area of Health science, in 2018. Specialty field is physical therapy. He has published 13 scientific papers.

Hitomi Takahashi graduated from Saitama Prefectural University in 2019. Therefore, she studied "Fall detection in toilet rooms using thermal imaging sensors." She is currently working for the Department of Physical Therapy, Ageo Central General Hospital. 